Research Article

\title{
Adaptive Neural Network Control Scheme of Switched Systems with Input Saturation
}

\author{
Xiaoli Jiang $\mathbb{D}^{1},{ }^{1}$ Mingyue Liu, ${ }^{1}$ Siqi Liu, ${ }^{1}$ Jing $\mathrm{Xu},{ }^{1}$ and Lina Liu $\mathbb{D}^{2}$ \\ ${ }^{1}$ College of Mathematics and Automation Research Institute, Bohai University, Jinzhou, Liaoning 121013, China \\ ${ }^{2}$ School of Electronic and Information Engineering, Soochow University, Suzhou, Jiangsu 215006, China \\ Correspondence should be addressed to Xiaoli Jiang; jxls309@163.com and Lina Liu; 1ln@suda.edu.cn
}

Received 29 June 2020; Revised 14 August 2020; Accepted 28 August 2020; Published 10 September 2020

Academic Editor: Lifei Chen

Copyright (c) 2020 Xiaoli Jiang et al. This is an open access article distributed under the Creative Commons Attribution License, which permits unrestricted use, distribution, and reproduction in any medium, provided the original work is properly cited.

This paper investigates a scheme of adaptive neural network control for a stochastic switched system with input saturation. The unknown smooth nonlinear functions are approximated directly by neural networks. A modified approach is proposed to deal with unknown functions with nonstrict feedback form in the design process. Furthermore, by combining the auxiliary design signal and the adaptive backstepping design, a valid adaptive neural tracking controller design algorithm is presented such that all the signals of the switched closed-loop system are in probability semiglobally, uniformly, and ultimately bounded, and the tracking error eventually converges to a small neighborhood of the origin in probability. In the end, the effectiveness of the proposed method is verified by a simulation example.

\section{Introduction}

Switched systems as a class of hybrid systems is made up of a race of continuous or discrete subsystems and switching rules [1-4]. In the last decades, switched systems have attracted more and more attention due to their significance in the modeling of many engineering applications, such as chemical processes, robot manipulators, and power systems. So far, many remarkable achievements about the stability analysis and synthesis have been made in the field of switched systems. In general, there are two ways of dealing with it: one is to find a common Lyapunov function to ensure stability of the switched systems under arbitrary switching laws; the other is to use a multiple Lyapunov functional technique to stabilize the switched systems under some designed switching laws, see, e.g., [5-10] and the references therein.

Almost all feedback controls are involved in saturation, due to the limitation of maximum and minimum values, and the sensors of actuators are saturated. If the controller is designed without considering input saturation, it can degrade the performance of the control system and even lead to instability. In recent years, the problem of input saturation limitation has attracted wide attention, and many methods for designing controllers have been proposed. Among them, PID controller was an earlier antisaturation method [11]. Workman designed a time optimal controller to reduce input saturation. Later, scholars proposed a saturation compensator, for example, the CNF controller was designed by $\mathrm{Hu}$ et al. [12], Grimm et al. [13], and Mulder et al. Mulder et al. [14] gave antisaturation compensation controllers by using linear matrix inequalities. However, there are still many control questions about switched systems with input saturation to be studied in depth.

According to recent research, the switching strategy plays an important role in the switching process. Due to the switching effect, the originally linear characteristics will become nonlinear. In addition, the features of multiple subsystems vary greatly and interact with each other and further increase the complexity and make the whole system show complex dynamic behavior. Therefore, how to choose the switching strategy and ensure the stability of the whole system is a hot topic at present. The adaptive backstepping technology can effectively solve the stabilization and tracking control [15-33]. Such as in [26], an output feedback scheme based on discrete adaptive approximation was 
proposed for uncertain switched stochastic interconnected systems, and the unknown nonlinear function was modeled by combining a fuzzy logic system. For stochastic systems with output constraints, an adaptive neural controller based on approximation was constructed [27]. Niu et al. [28] investigate the global adaptive control problem for a class of switched uncertain nonlinear systems. By improving modedependent average dwell time (MDADT) method, a new adaptive control scheme is established which ensures the global bounded. It should be noted that an adaptive tracking controller based on neural networks was considered for the single input switching system of lower-triangular structure in $[29,34]$. As for the switching systems, because it contains multiple subsystems, its motion pattern is richer and more complex than the previous single model. In all dynamic behaviors, there may be good performance, bad performance, or even unstable dynamics. Therefore, on the premise of ensuring the stability of the system, this work attempts to design an appropriate control strategy to make the system achieve better switching effect and optimized performance index.

Moreover, as it is known to all, neural networks (NNs) have strong approximation ability, which can be used to deal with the unknown nonlinear functions, and it can be discovered in many literatures [35-42]. Recently, the authors in [36] gave an adaptive scheme using multilayer neural networks. State feedback strategy was firstly investigated for nonaffine systems through neural network approximation [39]. Neural network output feedback controller has been designed in [42] for a large-scale stochastic systems. For nonstrict feedback systems, a dynamic surface method based on observer was established in [43]. However, the above control results were obtained under a conservative condition, which the considered system structures are always in strict feedback form. The drift functions may be a mixture of all state variables, which makes the systems be in the nonstrict feedback. Therefore, we aim to propose an adaptive neural networks scheme for a switched system with nonstrict feedback form. Different from the relevant literatures, we construct a new scheme of stochastic systems with input saturation and design switching strategies to resist the saturation.

Based on the above research progress, this work tries to establish a neural network framework for a stochastic switched system with input saturation. Compared with the existing literatures, the innovation points of this paper are stated as follows:

(i) Applying neural networks technique, a modified backstepping algorithm for a stochastic switched system in lower-triangular is established. A novel controller is constructed to track the unknown states.

(ii) It needs to adopt the Itô formula for stochastic system, which makes it difficult to structure Lyapunov function corresponding to gradient term and high-order Hessen term. To solve this problem, we successfully developed neural networks to stochastic switching situation, which reduces the conservativeness caused by feedback form when the input saturation happens.
The other parts of this article are organized as follows. In Section 2, we introduce some assumptions and preliminaries and describe problem statements. Furthermore, we give the neural control process and stability analysis in this section. The simulation results are shown in Section 3, and the conclusions are analyzed in Section 4.

\section{Preliminaries and Controller Design}

In this section, the switched system and its descriptions are formulated in Section 2.1, and some primary lemmas and definitions including stability theorem and Young's inequality are also described. In Section 2.2, we construct the adaptive neural controller and state the stability analysis.

2.1. Problem Descriptions. Now, let us first present some lemmas, definitions, and assumptions, and they will be used in the subsequent developments. Consider a stochastic switched system with nonstrict feedback form:

$$
\left\{\begin{array}{l}
\mathrm{d} \xi_{i}=F_{\sigma(t), i}\left(\bar{\xi}_{i}, \xi_{i+1}, \xi\right) \mathrm{d} t+\psi_{\sigma(t), i}^{T}(\xi) \mathrm{d} w, \quad 1 \leq i \leq n-1 \\
\mathrm{~d} \xi_{n}=F_{\sigma(t), n}\left(\bar{\xi}_{n}, u, \xi\right) \mathrm{d} t+\psi_{\sigma(t), n}^{T}(\xi) \mathrm{d} w \\
\eta=\xi_{1}
\end{array}\right.
$$

where $\quad \bar{\xi}_{i}=\left[\xi_{1}, \xi_{2}, \ldots, \xi_{i}\right]^{T} \in R, i=1,2, \ldots, N$, and $\xi=\left[\xi_{1}, \xi_{2}, \ldots, \xi_{n}\right]^{T} \in R^{n}$ and $\eta$ are the state and the output of the system, respectively. Let random function $\psi_{\sigma(t), i}(\xi)$ be smooth, and the switching signal is denoted by $\sigma(t)$ : $[0,+\infty) \longrightarrow I_{q}=1,2, \ldots, q$. For $\forall i=1,2, \ldots, n, p \in I_{q}$, assume that $f_{p, i}^{q}(\cdot)$ and $h_{p, i}$ are assumed to be unknown and locally Lipschitz functions. The unknown system functions $g_{\sigma(t), i}\left(\bar{\xi}_{i}\right), f_{\sigma(t), i}(\xi)$, and $h_{\sigma(t), i}(\xi)$ are contained in the function $F_{\sigma(t), i}$ and satisfy $g_{\sigma(t), i}\left(\bar{\xi}_{i}\right) \xi_{i+1}+f_{\sigma(t), i}(\xi)+$ $h_{\sigma(t), i}(\xi)=F_{\sigma(t), i}\left(\bar{\xi}_{i}, \xi_{i+1}, \xi\right)$ and $u(v)+f_{\sigma(t), n}(\xi)+h_{\sigma(t), n}(\xi)$ $=F_{\sigma(t), n}\left(\bar{\xi}_{i}, u, \xi\right)$, which are smooth and $f_{p, i}(0)=0$. The variate $\omega$ is an independent $r$-dimensional standard Wiener process. It is important to note that there is a regular convention for switching systems, solutions are continuous everywhere, and the states do not jump at the switching moment. Let $u(v)$ be the controller with input saturation, which can be written as

$$
u(v(t))=\operatorname{sat}(v(t))= \begin{cases}\operatorname{sign}(v(t)) \varepsilon_{0}, & |v(t)| \geq \varepsilon_{0}, \\ v(t), & |v(t)|<\varepsilon_{0} .\end{cases}
$$

Lemma 1 (see [43]). For each pair $(x, y) \in R^{2}$, Young's inequality holds

$$
x y \leq \frac{\varepsilon^{a}}{a}|x|^{a}+\frac{1}{b \varepsilon^{b}}|y|^{b},
$$

where $\varepsilon>0, a>1, b>1,(a-1)(b-1)=1$.

Assumption 1. The constants $h_{i 0}$ satisfy $\left|h_{i}(\xi)\right| \leq\left|h_{i 0}\right|$, $i=1,2, \ldots, n$. Consider the following stochastic system: 


$$
\mathrm{d} \xi(t)=f(\xi(t)) \mathrm{d} t+g(\xi(t)) \mathrm{d} w,
$$

where $\xi \in R^{n}$ is the system state, $\omega$ is a standard Wiener process, and $f: R^{n} \longrightarrow R^{n}$ and $g: R^{n} \longrightarrow R^{n}$ are locally Lipschitz functions and satisfy $f(0)=g(0)=0$.

Definition 1 (see [44]). For any given $V(\xi) \in C^{2}$, which is associated with the stochastic system (4), the infinitesimal generator $\mathscr{L}$ is defined as follows:

$$
\mathscr{L} V(\xi)=\frac{\partial V}{\partial x} f(\xi)+\frac{1}{2} \operatorname{Tr}\left\{g(\xi)^{T} \frac{\partial^{2} V}{\partial x^{2}} g(\xi)\right\},
$$

where $\operatorname{Tr}(A)$ is the trace of a matrix $A$.

Definition 2. The trajectory $\xi(t)$ of system (4) is said to be semiglobally uniformly ultimately bounded in $p$ th moment if, for some compact set $\Omega \in R^{n}$ and any initial state $\xi_{0}=\xi\left(t_{0}\right)$, there exist constant $\varepsilon>0$ and time constant $T=T\left(\varepsilon, \xi_{0}\right)$, such that $E\left[|\xi(t)|^{p}\right]<\varepsilon$ for all $t>t_{0}+T$, $p \in I_{q}=\{1,2, \ldots, q\}$. In particular, when $p=2$, it is usually called semiglobally, uniformly, and ultimately bounded in mean square.

Lemma 2 (see [45]). Consider the stochastic system (4). If there exist functions $V(\xi) \in C^{2}, \bar{\sigma}_{1}$, and $\bar{\sigma}_{2} \in K_{\infty}$ and constants $m_{0}>0$ and $n_{0}>0$, such that

$$
\begin{gathered}
\bar{\sigma}_{1}(\xi) \leq V(\xi) \leq \bar{\sigma}_{2}(\xi), \\
L V(\xi) \leq-m_{0} V(\xi)+n_{0},
\end{gathered}
$$

then, there is a unique solution of system (4) for each $\xi_{0} \in R^{n}$, and it holds

$$
E[V(\xi)] \leq V\left(\xi_{0}\right) e^{-m_{0} t}+\frac{m_{0}}{n_{0}}, \quad \forall t>t_{0}
$$

In this paper, approximation-based NNs will be used to approximate the unknown nonlinear functions. For any continuous unknown smooth nonlinear function $f(Z)$ over a compact set $\Omega_{Z} \subset R^{q}$, there exists NNs $W^{* T} S(Z)$, such that, for a desired level of accuracy $\varepsilon$,

$$
\begin{aligned}
f(Z) & =W^{* T} S(Z)+\delta(Z), \\
|\delta(Z)| & \leq \varepsilon,
\end{aligned}
$$

where $W^{*}$ is the ideal constant weight vector and defined by

$$
W^{*}=\arg \min _{W \in R^{N}}\left\{\sup _{Z \in \Omega_{Z}}\left|f(Z)-W^{T} S(Z)\right|\right\},
$$

$\delta(Z)$ is the approximation error, $W=\left[w_{1}, \ldots, w_{N}\right]^{T}$ is the weight vector, and $S(Z)=\left[s_{1}(Z), \ldots, s_{N}(Z)\right]^{T}$ is the radial basis function vector with $N$ being the number of the NNs nodes and $N>1$ :

$$
s_{i}(Z)=\exp \left[-\frac{\left(Z-u_{i}\right)^{T}\left(Z-u_{i}\right)}{\eta_{i}^{2}}\right], \quad i=1,2, \ldots, N
$$

where $u_{i}=\left[u_{i 1}, u_{i 2}, \ldots, u_{i n}\right]^{T}$ is the center of the receptive field and $\eta_{i} \in R$ is the width of the Gaussian function.

2.2. Adaptive Controller Design and Stability Analysis. A neural controller will be constructed for the stochastic switched system (1). At the same time, the adaptive laws will be given in this section.

At first, let us define some essential functions:

$$
\begin{aligned}
& \alpha_{1}=-k_{1} z_{1}-\frac{9}{4} z_{1}-\frac{z_{1}^{3} \Theta_{1}}{4 \tau \psi_{1}^{T}\left(\xi_{1}, \dot{\eta}_{d}\right) \psi_{1}\left(\xi_{1}, \dot{\eta}_{d}\right)}, \\
& \dot{\Theta}_{1}=\frac{z_{1}^{6} \gamma_{1}}{4 \tau \psi_{1}^{T}\left(\xi_{1}, \dot{\eta}_{d}\right) \psi_{1}\left(\xi_{1}, \dot{\eta}_{d}\right)}-\sigma_{1} \Theta_{1},
\end{aligned}
$$

where $\alpha_{1}$ is the first virtual control signal and $\dot{\Theta}_{1}$ is an adaptive law with $k_{1}, \tau, \gamma_{1}$, and $\sigma_{1}$ being positive design parameters.

Similarly, for $i=2, \ldots, n-1$, we design the virtual signals and adaptive laws as follows:

$$
\begin{aligned}
\alpha_{i} & =-k_{i} z_{i}-\frac{13}{4} z_{i}-\frac{z_{i}^{3} \Theta_{i}}{4 \tau \psi_{i}^{T}\left(\bar{\xi}_{i}, m_{i}\right) \psi_{i}\left(\bar{\xi}_{i}, m_{i}\right)}, \\
\dot{\Theta}_{i} & =\frac{z_{i}^{6} \gamma_{i}}{4 \tau \psi_{i}^{T}\left(\bar{\xi}_{i}, m_{i}\right) \psi_{i}\left(\bar{\xi}_{i}, m_{i}\right)}-\sigma_{i} \Theta_{i},
\end{aligned}
$$

where $m_{i}=\left[\eta_{d}, \dot{\eta}_{d}, \ldots, \eta_{d}^{(i-1)}, \widehat{\Theta}_{1}, \ldots, \widehat{\Theta}_{i-1}\right], \alpha_{i}$ is the $i$ th virtual control signal and $\Theta_{i}$ is the adaptive law with $k_{i}, \tau, \gamma_{i}$, and $\sigma_{n}$ being positive design parameters. The actual control input $v$ and the adaptive law $\dot{\Theta}_{n}$ are defined as follows:

$$
\begin{aligned}
v & =-k_{n} z_{n}-\frac{5}{2} z_{n}-\frac{z_{n}^{3} \Theta_{n}}{4 \tau \psi_{n}^{T}\left(\xi, m_{n}\right) \psi_{n}\left(\xi, m_{n}\right)}-\tilde{h}, \\
\dot{\Theta}_{n} & =\frac{z_{n}^{6} \psi_{n}}{4 \tau \psi_{n}^{T}\left(\xi, m_{n}\right) \psi_{n}\left(\xi, m_{n}\right)}-\sigma_{n} \Theta_{n},
\end{aligned}
$$

where $m_{n}=\left[\eta_{d}, \dot{\eta}_{d}, \ldots, \eta_{d}^{(i-1)}, \widehat{\Theta}_{1}, \ldots, \widehat{\Theta}_{i-1}\right], k_{n}, \tau, \gamma_{n}$, and $\sigma_{n}$ being positive design parameters. $\widetilde{h}$ is an auxiliary design signal, which will be defined later. The coordinate transformations are as follows:

$$
\begin{aligned}
& z_{i}=\xi_{i}-\alpha_{i-1}, \quad i=1,2, \ldots, n-1, \\
& z_{n}=\xi_{n}-\alpha_{n-1}-\tilde{h}
\end{aligned}
$$

where $\alpha_{0}=\eta_{d}(t)$. Combining (1) with coordinate transformations (17) and (18), the following system can be obtained: 


$$
\begin{aligned}
& \mathrm{d} z_{i}=\left(g_{i, p}\left(\bar{\xi}_{i}\right) \xi_{i+1}+f_{i, p}(\xi)+h_{i, p}(\xi)-l \alpha_{i-1}\right) \mathrm{d} t+\left(\psi_{i, p}(\xi)-\sum_{j=1}^{i-1} \frac{\partial \alpha_{i-1}}{\partial x_{j}} \psi_{j, p}^{T}(\xi)\right)^{T} \mathrm{~d} w \\
& \mathrm{~d} z_{n}=\left(u(v)+f_{n, p}(\xi)+h_{n, p}(\xi)-l \alpha_{n-1}-\dot{\tilde{h}}\right) \mathrm{d} t+\left(\psi_{n, p}(\xi)-\sum_{j=1}^{n-1} \frac{\partial \alpha_{n-1}}{\partial x_{j}} \psi_{j, p}^{T}(\xi)\right)^{T} \mathrm{~d} w
\end{aligned}
$$

where

$$
\begin{aligned}
l \alpha_{i-1}= & \sum_{j=1}^{i-1} \frac{\partial \alpha_{i-1}}{\partial \widehat{\Theta}_{j}} \dot{\Theta}_{j}+\sum_{j=1}^{i-1} \frac{\partial \alpha_{i-1}}{\partial \xi_{j}}\left(g_{j, p}\left(\bar{\xi}_{j}\right) \xi_{j+1}+f_{j, p}(\xi)+h_{j, p}(\xi)\right) \\
& +\sum_{j=1}^{i-1} \frac{\partial \alpha_{i-1}}{\partial \eta_{d}^{(j)}} \eta_{d}^{(j+1)}+\left(\sum_{m, n=1,1}^{i-1} \frac{1}{2} \frac{\partial^{2} \alpha_{i-1}}{\partial \xi_{m} \partial \xi_{n}} \psi_{m, p}^{T}(\xi) \psi_{n, p}(\xi)\right),
\end{aligned}
$$

with $l \alpha_{0}=\dot{\eta}_{d}$ for $i=2, \ldots, n$.

Theorem 1. Let Assumption 1 hold, the closed-loop structure be consisted of the virtual control signals in (11) and (13), the adaptive laws (12) and (14) together with (16) and the actual control signal (15), which are designed from the stochastic switched system (1). All signals are bounded with some suitable parameters and the tracking error enters inside the area $\Omega_{1}$ for all $t>T_{1}$ :

$$
\Omega_{1}=\left\{\eta(t) \in \frac{R}{E}\left[\left|\eta-\eta_{d}\right|^{4}\right] \leq 8 \frac{D_{n}}{d_{0}}, \forall_{t}>T_{1}\right\},
$$

where $d_{0}=\min \left\{4 k_{1} \bar{g}_{1}, \ldots, 4 k_{n-1} \bar{g}_{n-1}, 4 k_{n}, \sigma_{i}, 1 \leq i \leq n\right\}$,

$$
D_{n}=\sum_{i=1}^{n}\left(\frac{a_{i}^{2}}{2}+\frac{\varepsilon_{i}^{* 4}}{4}+\frac{\sigma_{i} \Theta_{i}^{* 2}}{2 \gamma_{i}}\right)+n \tau+\sum_{i=1}^{n} \frac{h_{i 0}^{4}}{4 \bar{g}_{i}^{4}}+\frac{h_{n 0}^{4}}{4},
$$

and $T_{1}=\max \left\{0,\left(1 / d_{0}\right) \ln \left(d_{0} v(0) / D_{n}\right)\right\}, \overline{g_{i}}=\inf \left\{g_{\sigma(t), i}\left(\bar{\xi}_{i}\right)\right\}$, and $\overline{g_{i}}>0$.

\section{Proof}

Step 1: choose the following Lyapunov function:

$$
V_{1}=\frac{1}{4 \bar{g}_{1}} z_{1}^{4}+\frac{1}{2 \gamma_{1}^{2}} \widetilde{\Theta}_{1}^{2},
$$

where $\bar{g}_{1}>0$ and $\gamma_{1}>0$ are design parameters, and $\widetilde{\Theta}_{1}=\Theta_{1}^{*}-\Theta_{1}$ with $\Theta_{1}$ being the estimation of $\Theta_{1}^{*}$. In the light of the above equations in (4), (5), and (19), it yields

$$
\begin{aligned}
L V_{1}= & \frac{1}{\bar{g}_{1}} z_{1}^{3}\left(g_{1, p}\left(\xi_{1}\right) \xi_{2}+f_{1, p}(\xi)+h_{1, p}(\xi)-\dot{\eta}_{d}\right) \\
& +\frac{3}{2 \bar{g}_{1}} z_{1}^{2} \psi_{1, p}^{T}(\xi)-\frac{1}{\gamma} \widetilde{\Theta}_{1} \dot{\Theta}_{1} .
\end{aligned}
$$

Employing Young's inequality (3), we can obtain

$$
\begin{aligned}
& \frac{3}{2 \bar{g}_{1}} z_{1}^{2} \psi_{1, p}^{T}(\xi) \psi_{1, p}(\xi) \leq \frac{3}{2 \bar{g}_{1}} z_{1}^{2}\left\|\psi_{1, p}(\xi)\right\|^{2} \\
& \leq \frac{1}{a_{1} \bar{g}_{1}^{3 / 2}} z_{1}^{3}\left\|\psi_{1, p}(\xi)\right\|^{3}+\frac{a_{1}^{2}}{2}, \\
& \frac{1}{\bar{g}_{1}} z_{1}^{3} h_{1, p}(\xi) \leq \frac{3}{4} z_{1}^{4}+\frac{1}{4 \bar{g}_{1}^{4}} h_{10}^{4},
\end{aligned}
$$

where $a_{1}$ is a positive constant. The NNs $\omega_{1}^{*}{ }_{p}^{T} \phi_{1}\left(\xi, \dot{\eta}_{d}\right)$ can estimate the following unknown function:

$$
\begin{aligned}
\frac{1}{\bar{g}_{1}} f_{1}(\xi)-\frac{1}{\bar{g}_{1}} \dot{\eta}_{d}+\frac{1}{a_{1} \bar{g}_{1}^{3 / 2}}\left\|\psi_{1, p}(\xi)\right\|^{3}= & \omega_{1}^{*}{ }_{p}^{T} \phi_{1}\left(\xi, \dot{\eta}_{d}\right) \\
& +\delta_{1}\left(\xi, \dot{\eta}_{d}\right) .
\end{aligned}
$$

According to (17), (25), and (27), formula (24) can be rewritten as

$$
\begin{aligned}
L V_{1} \leq & z_{1}^{3}\left(z_{2}+\frac{3}{4} z_{1}+\alpha_{1}+\omega_{1}^{*},{ }_{p}^{T} \phi_{1}\left(\xi, \dot{\eta}_{d}\right)+\delta_{1}\left(\xi, \dot{\eta}_{d}\right)\right) \\
& +\frac{a_{1}^{2}}{2}+\frac{1}{4 \bar{g}_{1}^{4}} h_{10}^{4}-\frac{1}{\gamma_{1}} \widetilde{\Theta}_{1} \dot{\Theta}_{1} .
\end{aligned}
$$

Therefore, the following result can be obtained:

$$
\begin{aligned}
z_{1}^{3} \omega_{1}^{*},{ }_{p}^{T} \phi_{1}\left(\xi, \dot{\eta}_{d}\right) & \leq \frac{z_{1}^{6} \omega_{1}^{*},{ }_{p}^{T} \phi_{1}^{T}\left(\xi, \dot{\eta}_{d}\right) \phi_{1}\left(\xi, \dot{\eta}_{d}\right)}{4 \tau}+\tau \\
& \leq \frac{z_{1}^{6}\left\|w_{1, p}^{*}\right\|^{2} \phi_{1}^{T}\left(\xi, \dot{\eta}_{d}\right) \phi_{1}\left(\xi, \dot{\eta}_{d}\right)}{4 \tau \phi_{1}^{T}\left(\xi, \dot{\eta}_{d}\right) \phi_{1}\left(\xi, \dot{\eta}_{d}\right)}+\tau \\
& \leq \frac{z_{1}^{6} \Theta_{1}^{*}}{4 \tau \phi_{1}^{T}\left(\xi, \dot{\eta}_{d}\right) \phi_{1}\left(\xi, \dot{\eta}_{d}\right)}+\tau, \\
z_{1}^{3} \delta_{1}\left(\xi, \dot{\eta}_{d}\right) & \leq \frac{3}{4} z_{1}^{4}+\frac{1}{4} \delta_{1}^{* 4}, \\
z_{1}^{3} z_{2} & \leq \frac{3}{4} z_{1}^{4}+\frac{1}{4} z_{2}^{4},
\end{aligned}
$$

where $\Theta_{1}^{*}=\max \left\{\left\|\omega_{1, p}^{*}\right\|^{2}: p \in I_{p}\right\},\left|\delta_{1}\left(\xi, \dot{\eta}_{d}\right)\right| \leq \delta_{1}^{*}, \delta_{1}^{*}$ and $\tau$ are positive constants, and 


$$
0<\phi_{1}^{T}\left(\xi, \dot{\eta}_{d}\right) \phi_{1}\left(\xi, \dot{\eta}_{d}\right) \leq 1 .
$$

Substituting (29)-(31) into (28), we have

$$
\begin{aligned}
L V_{1} \leq & z_{1}^{3}\left(\frac{9}{4} z_{1}+\alpha_{1}+\frac{z_{1}^{3} \Theta_{1}}{4 \tau \phi_{1}^{T}\left(\xi, \dot{\eta}_{d}\right) \phi_{1}\left(\xi, \dot{\eta}_{d}\right)}\right) \\
& +\frac{z_{1}^{6} \widetilde{\Theta}_{1}}{4 \tau \phi_{1}^{T}\left(\xi, \dot{\eta}_{d}\right) \phi_{1}\left(\xi, \dot{\eta}_{d}\right)}+\frac{a_{1}^{2}}{2}+\frac{1}{4 \bar{g}_{1}^{4}} h_{10}^{4} \\
& +\tau+\frac{1}{4} z_{2}^{4}+\frac{1}{4} \delta_{1}^{* 4}-\frac{1}{\gamma_{1}} \widetilde{\Theta}_{1} \dot{\Theta}_{1} .
\end{aligned}
$$

Substituting the virtual controller $\alpha_{1}$ in (11) and the adaptive law in (12) into (33) yields

$$
L V_{1} \leq-k_{1} z_{1}^{4}+\frac{\sigma_{1}}{\gamma_{1}} \widetilde{\Theta}_{1} \dot{\Theta}_{1}+\frac{1}{4} z_{2}^{4}+\frac{a_{1}^{2}}{2}+\frac{1}{4 \bar{g}_{1}^{4}} h_{10}^{4}+\tau+\frac{1}{4} \delta_{1}^{* 4} .
$$

We can get the following result by Young's inequality:

$$
\frac{\sigma_{1}}{\gamma_{1}} \widetilde{\Theta}_{1} \Theta_{1}=\frac{\sigma_{1}}{\gamma_{1}} \widetilde{\Theta}_{1}\left(\Theta_{1}^{*}-\widetilde{\Theta}_{1}\right) \leq-\frac{\sigma_{1}}{2 \gamma_{1}} \widetilde{\Theta}_{1}^{2}+\frac{\sigma_{1}}{2 \gamma_{1}} \widetilde{\Theta}_{1}^{* 2} .
$$

Then, one has

$$
L V_{1} \leq-k_{1} z_{1}^{4}-\frac{\sigma_{1}}{2 \gamma_{1}} \widetilde{\Theta}_{1}^{2}+\frac{1}{4} z_{2}^{4}+D_{1}
$$

where $D_{1}=\left(a_{1}^{2} / 2\right)+\tau+\left(\delta_{1}^{* 4} / 4\right)+\left(h_{10}^{4} / 4 \bar{g}_{1}^{4}\right)+\left(\sigma_{1} \Theta_{1}^{* 2} /\right.$ $\left.2 \gamma_{1}\right)$.

Step $i(i=2,3, \ldots, n-1)$ : similar to Step 1 , choose the following Lyapunov function:

$$
V_{i}=V_{i-1}+\frac{1}{4 \bar{g}_{i}} z_{i}^{4}+\frac{1}{2 \gamma_{i}} \widetilde{\Theta}_{i}^{2},
$$

where $\bar{g}_{i}$ and $\gamma_{i}$ are positive constants and $\Theta_{i}$ is the estimation of $\Theta_{i}^{*}, \widetilde{\Theta}_{i}=\Theta_{i}^{*}-\Theta_{i}$. Then, we can obtain the following formula:

$$
\begin{aligned}
L V_{i}= & L V_{i-1}+\frac{1}{\bar{g}_{i}} z_{i}^{3}\left(g_{i, p}\left(\bar{\xi}_{i}\right) \xi_{i+1}+f_{i, p}(\xi)+h_{i, p}(\xi)-\sum_{j=1}^{i-1} \frac{\partial \alpha_{i-1}}{\partial \xi_{j}}\left(g_{j, p}\left(\bar{\xi}_{j}\right) \xi_{j+1}+f_{j, p}(\xi)+h_{j, p}(\xi)\right)\right. \\
& \left.-\sum_{j=0}^{i-1} \frac{\partial \alpha_{i-1}}{\partial \eta_{d}^{(j)}} \eta_{d}^{(j+1)}-\sum_{j=1}^{i-1} \frac{\partial \alpha_{i-1}}{\partial \widehat{\Theta}_{j}} \dot{\Theta}_{j}-\frac{1}{\gamma_{1}} \widetilde{\Theta}_{i} \dot{\Theta}_{i}-\frac{1}{2} \sum_{m, n=1}^{i-1} \frac{\partial^{2} \alpha_{i-1}}{\partial \xi_{p} \partial \xi_{q}} \psi_{m, p}^{T}(\xi) \psi_{n, p}(\xi)\right) \\
& +\frac{3}{2 \bar{g}_{i}} z_{i}^{2}\left(\psi_{i, p}(\xi)-\sum_{j=1}^{i-1} \frac{\partial \alpha_{i-1}}{\partial \xi_{j}} \psi_{j, p}(\xi)\right)^{T}\left(\psi_{i, p}(\xi)-\sum_{j=1}^{i-1} \frac{\partial \alpha_{i-1}}{\partial \xi_{j}} \psi_{j, p}(\xi)\right),
\end{aligned}
$$

where

$$
\begin{aligned}
& \frac{3}{2 \bar{g}_{i}} z_{i}^{2}\left(\psi_{i, p}(\xi)-\sum_{j=1}^{i-1} \frac{\partial \alpha_{i-1}}{\partial \xi_{j}} \psi_{j, p}(\xi)\right)^{T}\left(\psi_{i, p}(\xi)-\sum_{j=1}^{i-1} \frac{\partial \alpha_{i-1}}{\partial \xi_{j}} \psi_{j, p}(\xi)\right) \\
& \quad \leq \frac{3}{2 \bar{g}_{i}} z_{2}^{2}\left\|\psi_{i, p}(\xi)-\sum_{j=1}^{i-1} \frac{\partial \alpha_{i-1}}{\partial \xi_{j}} \psi_{j, p}(\xi)\right\|^{2} \\
& \leq \frac{z_{i}^{3}}{a_{i} \bar{g}_{i}^{3 / 2}}\left\|\psi_{i, p}(\xi)-\sum_{j=1}^{i-1} \frac{\partial \alpha_{i-1}}{\partial \xi_{j}} \psi_{j, p}(\xi)\right\|^{3}+\frac{a_{i}^{2}}{2}
\end{aligned}
$$

where $a_{i}$ is a positive constant. By Young's inequality, one has

$$
\begin{gathered}
\frac{1}{\bar{g}_{i}} z_{i}^{3} h_{i, p}(\xi) \leq \frac{3}{4} z_{i}^{4}+\frac{1}{4 \bar{g}_{i}^{4}} h_{i 0}^{4}, \\
-\frac{1}{\bar{g}_{i}} z_{i}^{3} \sum_{j=1}^{i-1} \frac{\partial \alpha_{i-1}}{\partial \xi_{j}} h_{j, p}(\xi) \leq \frac{1}{4 \bar{g}_{i}^{4}} \sum_{j=1}^{i-1}\left(\frac{\partial \alpha_{i-1}}{\partial \xi_{j}}\right)^{4} h_{j 0}^{4}+\frac{3}{4} z_{i}^{4} .
\end{gathered}
$$

Then, we apply NNs $\omega_{i}^{*},{ }_{p}^{T} \phi_{i}\left(\xi, m_{i}\right)$ to replace the unknown function:

$$
\begin{aligned}
& w_{i}^{*},_{p} \phi_{i}\left(\xi, m_{i}\right)+\delta_{i}\left(\xi, m_{i}\right) \\
& =\frac{1}{\bar{g}_{j}}\left\{f_{i, p}(\xi)-\sum_{j=1}^{i-1} \frac{\partial \alpha_{i-1}}{\partial \xi_{j}}\left(g_{j, p}\left(\bar{\xi}_{j}\right) \xi_{j+1}+f_{i, p}(\xi)\right)\right. \\
& \quad-\sum_{j=0}^{i-1} \frac{\partial \alpha_{i-1}}{\partial \eta_{d}^{(j)}} \eta_{d}^{(j+1)}-\sum_{j=1}^{i-1} \frac{\partial \alpha_{i-1} \widehat{\Theta}_{j}}{\partial \widehat{\Theta}_{j}} \\
& \left.\quad-\frac{1}{2} \sum_{m, n=1}^{i-1} \frac{\partial^{2} \alpha_{i-1}}{\partial \xi_{m} \partial \xi_{n}} \psi_{m, p}^{T}(\xi) \psi_{n, p}(\xi)\right\} \\
& \quad+\frac{1}{a_{i} \bar{g}_{i}^{3 / 2}}\left\|\psi_{i, p}(\xi)-\sum_{j=1}^{i-1} \frac{\partial \alpha_{i-1}}{\partial \xi_{j}} \psi_{j, p}(\xi)\right\|^{3} \\
& +\frac{1}{a_{i} \bar{g}^{4}} \sum_{j=1}^{i-1}\left(\frac{\partial \alpha_{i-1}}{\partial \xi_{j}}\right)^{4} h_{j 0}^{4} .
\end{aligned}
$$


Due to (17) and (39)-(42), we have

$$
\begin{aligned}
L V_{i} \leq & L V_{i-1}+z_{i}^{3}\left(z_{i+1}+\frac{3}{2} z_{i}+w_{i}^{*}{ }_{p}^{T} \phi_{i}\left(\xi, m_{i}\right)+\delta_{i}\left(\xi, m_{i}\right) \alpha_{i}\right) \\
& +\frac{a_{i}^{2}}{2}+\frac{1}{4 \bar{g}_{i}^{4}} h_{10}^{4}-\frac{1}{\gamma_{i}} \widetilde{\Theta}_{i} \dot{\Theta}_{i} .
\end{aligned}
$$

In line with Young's inequality (3), one can obtain

$$
\begin{aligned}
z_{i}^{3} w_{i}^{*}{ }_{p}^{T} \phi_{i}\left(\xi, m_{i}\right) & \leq \frac{z_{i}^{6} w_{i}^{*},{ }_{p}^{T} w_{i, p}^{*} \phi_{i}^{T}\left(\xi, m_{i}\right) \phi_{i}\left(\xi, m_{i}\right)}{4 \tau}+\tau \\
& \leq \frac{z_{i}^{6}\left\|w_{i, p}^{*}\right\|^{2} \phi_{i}^{T}\left(\xi, m_{i}\right) \phi_{i}\left(\xi, m_{i}\right)}{4 \tau \phi_{i}^{T}\left(\bar{\xi}_{i}, m_{i}\right) \phi_{i}\left(\xi, m_{i}\right)}+\tau \\
& \leq \frac{z_{i}^{6} \Theta_{i}^{*}}{4 \tau \phi_{i}^{T}\left(\bar{\xi}_{i}, m_{i}\right) \phi_{i}\left(\bar{\xi}_{i}, m_{i}\right)}+\tau,
\end{aligned}
$$

$$
\begin{aligned}
z_{i}^{3} \delta_{i}\left(\xi, m_{i}\right) & \leq \frac{3}{4} z_{i}^{4}+\frac{1}{4} \delta_{i}^{* 4}, \\
z_{i}^{3} z_{2} & \leq \frac{3}{4} z_{i}^{4}+\frac{1}{4} z_{i+1}^{4},
\end{aligned}
$$

where $\Theta_{i}^{*}=\max \left\{\left\|\omega_{i, p}^{*}\right\|^{2}: p \in I_{p}\right\}, \delta_{i}\left(\xi, m_{i}\right) \leq \delta_{i}^{*}$,

$$
0<\phi_{i}^{T}\left(\xi, m_{i}\right) \phi_{i}\left(\xi, m_{i}\right) \leq 1,
$$

$\delta_{i}^{*}$ and $\tau$ are positive constants. Substituting (44)-(46) into (43), we can obtain

$$
\begin{aligned}
& \begin{array}{r}
L V_{i} \leq z_{i}^{3}\left(3 z_{i}+\alpha_{i}+\frac{z_{i}^{3} \Theta_{i}^{*}}{4 \tau \phi_{i}^{T}\left(\bar{\xi}_{i}, m_{i}\right) \phi_{i}\left(\bar{\xi}_{i}\right.}\right. \\
\leq \sum_{m=1}^{i-1} k_{m} z_{m}^{4}-\sum_{m=1}^{i-1} \frac{\sigma_{m}}{2 \gamma_{m}} \widetilde{\Theta}_{m}^{2}+\frac{1}{4} z_{i+1}^{4} \\
+\frac{z_{i}^{6} \widetilde{\Theta}_{i}}{4 \tau \phi_{i}^{T}\left(\bar{\xi}_{i}, m_{i}\right) \phi_{i}\left(\bar{\xi}_{i}, m_{i}\right)}+\frac{\alpha_{i}^{2}}{2} \\
\text { re } D_{i-1}=\sum_{j=1}^{i-1}\left(\left(a_{j}^{2} / 2\right)+\left(1 / 4 \bar{g}_{j}^{4}\right) h_{j 0}^{4}+(1 / 4) \delta_{j}^{* 4}+\right. \\
(i-1) \tau \text {. Based on the designed virtual control } \\
L V_{i} \leq-\sum_{m=1}^{i} k_{m} z_{m}^{4}-\sum_{m=1}^{i-1} \frac{\sigma_{m}}{2 \gamma_{m}} \widetilde{\Theta}_{m}^{2}+\frac{\sigma_{i}}{\gamma_{i}} \widetilde{\Theta}_{i} \Theta_{i}+\frac{a_{i}^{2}}{2}
\end{array} \\
& +\frac{1}{4 \bar{g}_{i}^{4}} h_{i 0}^{4}+\tau+\frac{1}{4} z_{i+1}^{4}+\frac{1}{4} \delta_{i}^{* 4}+D_{i-1} .
\end{aligned}
$$

where $D_{i-1}=\sum_{j=1}^{i-1}\left(\left(a_{j}^{2} / 2\right)+\left(1 / 4 \bar{g}_{j}^{4}\right) h_{j 0}^{4}+(1 / 4) \delta_{j}^{* 4}+\left(\sigma_{j} \Theta_{j}^{* 2} /\right.\right.$ $\left.\left.2 \gamma_{j}\right)\right)+(i-1) \tau$. Based on the designed virtual control signal $\alpha_{i}$ in (13) and the adaptive law $\dot{\Theta}_{i}$ in (14), one can obtain$$
\text { imilar to (23), we can derive the following conclusion: }
$$$$
\frac{\sigma_{i}}{\gamma_{i}} \widetilde{\Theta}_{i} \Theta_{i}=\frac{\sigma_{i}}{\gamma_{i}} \widetilde{\Theta}_{i}\left(\Theta_{i}^{*}-\widetilde{\Theta}_{i}\right) \leq-\frac{\sigma_{i}}{2 \gamma_{i}} \widetilde{\Theta}_{i}^{2}+\frac{\sigma_{i}}{2 \gamma_{i}} \widetilde{\Theta}_{i}^{* 2} \text {. }
$$

Moreover, substituting (50) into (49) implies

$$
L V_{i} \leq-\sum_{m=1}^{i} k_{m} z_{m}^{4}-\sum_{m=1}^{i} \frac{\sigma_{m}}{2 \gamma_{m}} \widetilde{\Theta}_{m}^{2}+\frac{1}{4} z_{i+1}^{4}+D_{i}
$$

where $D_{i}=\sum_{j=1}^{i}\left(\left(a_{j}^{2} / 2\right)+\left(1 / 4 \bar{g}_{j}^{4}\right) h_{j 0}^{4}+(1 / 4) \delta_{j}^{* 4}+\left(\sigma_{j} \Theta_{j}^{* 2} /\right.\right.$ $\left.\left.2 \gamma_{j}\right)\right)+i \tau$.

Step $n$ : the Lyapunov function is chosen as follows:

$$
V_{n}=V_{n-1}+\frac{1}{4 \bar{g}_{n}} z_{n}^{4}+\frac{1}{2 \gamma_{n}} \widetilde{\Theta}_{n}^{2}
$$

where $\bar{g}_{n}$ and $\gamma_{n}$ are positive constants and $\Theta_{n}$ is the estimation of $\Theta_{n}^{*}, \widetilde{\Theta}_{n}=\Theta_{n}^{*}-\Theta_{n}$. From Itô differential formula, we can obtain the following result: 
Discrete Dynamics in Nature and Society

7

$$
\begin{aligned}
L V_{n}= & L V_{n-1}+z_{n}^{3}\left(u(v)+f_{n, p}(\xi)+h_{n, p}(\xi)\right. \\
& -\sum_{j=1}^{n-1} \frac{\partial \alpha_{n-1}}{\partial \xi_{j}}\left(g_{j, p}\left(\bar{\xi}_{j}\right) \xi_{j+1}+f_{j, p}(\xi)+h_{j, p}(\xi)\right) \\
& -\sum_{j=0}^{n-1} \frac{\partial \alpha_{n-1}}{\partial \widehat{\theta}_{j}} \dot{\hat{\theta}}_{j}-\sum_{j=0}^{n-1} \frac{\partial \alpha_{n-1}}{\partial \eta_{d}^{(j)}} \eta_{d}^{(j+1)}-\dot{\widetilde{h}} \\
& \left.-\frac{1}{2} \sum_{m, n=1}^{n-1} \frac{\partial^{2} \alpha_{n-1}}{\partial \xi_{m} \partial \xi_{n}} \psi_{m, p}^{T}(\xi) \psi_{n, p}(\xi)\right)-\frac{1}{\gamma_{n}} \widetilde{\Theta}_{n} \dot{\Theta}_{n} \\
& +\frac{3 z_{n}^{2}}{2}\left(\psi_{n, p}(\xi)-\sum_{j=1}^{n-1} \frac{\partial \alpha_{n-1}}{\partial \xi_{j}} \psi_{j, p}(\xi)\right)^{T} \\
& \cdot\left(\psi_{n, p}(\xi)-\sum_{j=1}^{n-1} \frac{\partial \alpha_{n-1}}{\partial \xi_{j}} \psi_{j, p}(\xi)\right) .
\end{aligned}
$$

We introduce the following dynamic system in order to simplify the controller design:

$$
\dot{\widetilde{h}}=-\widetilde{h}+u(v)-v
$$

Based on (5), one has

$$
\begin{gathered}
z_{n}^{3} h_{n, p}(\xi) \leq \frac{3}{4} z_{n}^{4}+h_{n 0}^{4}, \\
-z_{n}^{3} \sum_{j=1}^{n-1} \frac{\partial \alpha_{n-1}}{\partial \xi_{j}} h_{j, p}(\xi) \leq \frac{1}{4} \sum_{j=1}^{n-1}\left(\frac{\partial \alpha_{n-1}}{\partial \xi_{j}}\right)^{4} h_{j 0}^{4}+\frac{3}{4} z_{n}^{4} .
\end{gathered}
$$

Similar to (39), the following inequality can be written as

$$
\begin{aligned}
& \frac{3}{2} z_{n}^{2}\left(\psi_{n, p}(\xi)-\sum_{j=1}^{n-1} \frac{\partial \alpha_{n-1}}{\partial \xi_{j}} \psi_{j, p}(\xi)\right)^{T} \\
& \cdot\left(\psi_{n, p}(\xi)-\sum_{j=1}^{n-1} \frac{\partial \alpha_{n-1}}{\partial \xi_{j}} \psi_{j, p}(\xi)\right) \\
& \leq \frac{3}{2} z_{n}^{2}\left\|\psi_{n, p}(\xi)-\sum_{j=1}^{n-1} \frac{\partial \alpha_{n-1}}{\partial \xi_{j}} \psi_{j, p}(\xi)\right\|^{2} \\
& \leq \frac{z_{n}^{3}}{a_{n}}\left\|\psi_{n, p}(\xi)-\sum_{j=1}^{n-1} \frac{\partial \alpha_{n-1}}{\partial \xi_{j}} \psi_{j, p}(\xi)\right\|^{3}+\frac{a_{n}^{2}}{2},
\end{aligned}
$$

where $a_{n}>0$. Substituting (54)-(57) into (53), we obtain

$$
\begin{aligned}
L V_{n}= & L V_{n-1}+z_{n}^{3}\left(\frac{3}{2} z_{n}+f_{n, p}(\xi)-\sum_{j=1}^{n-1} \frac{\partial \alpha_{n-1}}{\partial \xi_{j}}\left(g_{j, p}\left(\bar{\xi}_{j}\right) \xi_{j+1}+f_{j, p}(\xi)\right)\right. \\
& -\sum_{j=0}^{n-1} \frac{\partial \alpha_{n-1}}{\partial \eta_{d}^{(j)}} \eta_{d}^{(j+1)}-\sum_{j=1}^{n-1} \frac{\partial \alpha_{n-1}}{\partial \widehat{\Theta}_{j}} \dot{\Theta}_{j} \\
& -\frac{1}{2} \sum_{m, n=1}^{n-1} \frac{\partial^{2} \alpha_{n-1}}{\partial \xi_{p} \partial \xi_{q}} \psi_{m, p}^{T}(\xi) \psi_{n, p}(\xi) \\
& \left.+\widetilde{h}+v+\frac{1}{a_{n}}\left\|\psi_{n, p}(\xi)-\sum_{j=1}^{n-1} \frac{\partial \alpha_{n-1}}{\partial \xi_{j}} \psi_{j, p}(\xi)\right\|^{3}\right) \\
& +\frac{1}{4} \sum_{j=1}^{n-1}\left(\frac{\partial \alpha_{n-1}}{\partial \xi_{j}}\right)^{4} h_{j 0}^{4}+\frac{1}{4} h_{n 0}^{4}-\frac{1}{\gamma_{n}} \widetilde{\Theta}_{n} \dot{\Theta}_{n}+\frac{a_{n}^{2}}{2}
\end{aligned}
$$

The neural networks can estimate the unknown nonlinear function:

$$
\begin{aligned}
w_{n p p}^{* T} \phi_{n}\left(\xi, m_{n}\right)+\delta_{n}\left(\xi, m_{n}\right) & f_{i, p}(\xi)-\sum_{j=1}^{n-1} \frac{\partial \alpha_{i-1}}{\partial \xi_{j}}\left(g_{j, p}\left(\bar{\xi}_{j}\right) \xi_{j+1}+f_{i, p}(\xi)\right) \\
& -\sum_{j=0}^{n-1} \frac{\partial \alpha_{n-1}}{\partial \eta_{d}^{(j)}} \eta_{d}^{(j+1)}-\sum_{j=1}^{n-1} \frac{\partial \alpha_{n-1}}{\partial \widehat{\Theta}_{j}} \dot{\widehat{\Theta}}_{j}-\sum_{j=0}^{n-1} \frac{\partial \alpha_{n-1}}{\partial \eta_{d}^{(j)}} \eta_{d}^{(j+1)} \\
& -\sum_{j=1}^{n-1} \frac{\partial \alpha_{n-1}}{\partial \widehat{\Theta}_{j}} \dot{\widehat{\Theta}}_{j}-\frac{1}{2} \sum_{m, n=1}^{n-1} \frac{\partial^{2} \alpha_{n-1}}{\partial \xi_{p} \partial \xi_{q}} \psi_{m, p}^{T}(\xi) \psi_{n, p}(\xi) \\
& +\frac{1}{4} \sum_{j=1}^{n-1}\left(\frac{\partial \alpha_{n-1}}{\partial \xi_{j}}\right)^{4} h_{j 0}^{4}+\frac{1}{a_{n}}\left\|\psi_{n, p}(\xi)-\sum_{j=1}^{n-1} \frac{\partial \alpha_{n-1}}{\partial \xi_{j}} \psi_{j, p}(\xi)\right\|^{3} .
\end{aligned}
$$

Similarly, it is noted that the following formula holds:

$$
\begin{aligned}
z_{n}^{3} w_{n}^{*}{ }_{p}^{T} \phi_{n}\left(\xi, m_{n}\right) & \leq \frac{z_{n}^{6} w_{n, p}^{* T} \phi_{n}^{T}\left(\xi, m_{n}\right) \phi_{n}\left(\xi, m_{n}\right)}{4 \tau}+\tau \\
& \leq \frac{z_{n}^{6}\left\|w_{n, p}^{*}\right\|^{2} \phi_{n}^{T}\left(\xi, m_{n}\right) \phi_{n}\left(\xi, m_{n}\right)}{4 \tau \phi_{n}^{T}\left(\xi, m_{n}\right) \phi_{n}\left(\xi, m_{n}\right)}+\tau \\
& \leq \frac{z_{1}^{6} \Theta_{1}^{*}}{4 \tau \phi_{n}^{T}\left(\xi, m_{n}\right) \phi_{n}\left(\xi, m_{n}\right)}+\tau, \\
z_{n}^{3} \delta_{n}\left(\xi, m_{n}\right) & \leq \frac{3}{4} z_{n}^{4}+\frac{1}{4} \delta_{n}^{* 4},
\end{aligned}
$$


where $\delta_{n}^{*}$ and $\tau$ are positive constants, $\delta_{n}\left(\xi, m_{n}\right) \leq \delta_{n}^{*}$

$$
\begin{aligned}
0 & <\phi_{n}^{T}\left(\xi, m_{n}\right) \phi_{n}\left(\xi, m_{n}\right) \leq 1, \\
\Theta_{n}^{*} & =\max \left\{\left\|\omega_{n, p}^{*}\right\|^{2}: p \in I_{p}\right\} .
\end{aligned}
$$

Based on (59)-(61), the equality of (58) can be rewritten as

$$
\begin{aligned}
L V_{n} \leq & -\sum_{i=1}^{n-1} k_{i} z_{i}^{4}-\sum_{i=1}^{n-1} \frac{\sigma_{i}}{2 \gamma_{i}} \widetilde{\Theta}_{i}^{2} \\
& +z_{n}^{3}\left(\widetilde{h}+v+\frac{5}{2} z_{n}+\frac{z_{n}^{3} \Theta_{n}}{4 \tau \phi_{n}^{T}\left(\xi, m_{n}\right) \phi_{n}\left(\xi, m_{n}\right)}\right) \\
& +\frac{a_{n}^{2}}{2}+\tau+\frac{1}{4} \delta_{n}^{* 4}+D_{n-1}+\frac{1}{4} h_{n 0}^{4} \\
& +\frac{\widetilde{\Theta}_{n}}{\gamma_{n}}\left(\frac{z_{n}^{6} \gamma_{n}}{4 \tau \phi_{n}^{T}\left(\xi, m_{n}\right) \phi_{n}\left(\xi, m_{n}\right)}-\dot{\Theta}_{n}\right) .
\end{aligned}
$$

Taking formulas (15) and (16) into (63), it yields

$$
\begin{aligned}
L V_{n} \leq & -\sum_{i=1}^{n} k_{i} z_{i}^{4}-\sum_{i=1}^{n-1} \frac{\sigma_{i}}{2 \gamma_{i}} \widetilde{\Theta}_{i}^{2}+\frac{\sigma_{n}}{\gamma_{n}} \widetilde{\Theta}_{n} \Theta_{n} \\
& +\frac{a_{n}^{2}}{2}+\tau+\frac{1}{4} \delta_{n}^{* 4}+D_{n-1}+\frac{1}{4} h_{n 0}^{4},
\end{aligned}
$$

where

$$
\frac{\sigma_{n}}{\gamma_{n}} \widetilde{\Theta}_{n} \Theta_{n}=\frac{\sigma_{n}}{\gamma_{n}} \widetilde{\Theta}_{n}\left(\Theta_{n}^{*}-\widetilde{\Theta}_{n}\right) \leq-\frac{\sigma_{n}}{2 \gamma_{n}} \widetilde{\Theta}_{n}^{2}+\frac{\sigma_{n}}{2 \gamma_{n}} \Theta_{n}^{* 2} .
$$

In addition, substituting (65) into (64), we have

$$
\begin{aligned}
L V_{n} & \leq-\sum_{i=1}^{n} k_{i} z_{i}^{4}-\sum_{i=1}^{n} \frac{\sigma_{i}}{2 \gamma_{i}} \widetilde{\Theta}_{i}^{2}+\frac{\sigma_{n}}{2 \gamma_{n}} \Theta_{n}^{* 2}+\frac{a_{n}^{2}}{2}+\tau+\frac{1}{4} \varepsilon_{n}^{* 4}+D_{n-1} \\
& \leq-\sum_{i=1}^{n} k_{i} z_{i}^{4}-\sum_{i=1}^{n} \frac{\sigma_{i}}{2 \gamma_{i}} \widetilde{\Theta}_{i}^{2}+D_{n} .
\end{aligned}
$$

Then, (66) can be rewritten as

$$
L V_{n} \leq-d_{0} V_{n}+D_{n}
$$

where $\quad D_{n}=\sum_{i=1}^{n}\left(\left(a_{i}^{2} / 2\right)+(1 / 4) \varepsilon_{n}^{* 4}+\left(\sigma_{i} / 2 \gamma_{i}\right) \widetilde{\Theta}_{i}^{2}\right)+n \tau+$ $\sum_{i=1}^{n}\left(h_{i 0}^{4} / 4 \bar{g}_{i}^{4}\right)+\left(h_{i 0}^{4} / 4\right)$,

$$
d_{0}=\min \left\{4 k_{1} \bar{g}_{1}, \ldots, 4 k_{n-1} \bar{g}_{n-1}, 4 k_{n}, \sigma_{1}, \ldots, \sigma_{n}\right\} .
$$

Based on (63), we can easily obtain the following inequality:

$$
\frac{\mathrm{d} E[V(t)]}{\mathrm{d} t} \leq-d_{0} E[V(t)]+D_{n}
$$

Then, integrating inequality (69), the following formula can be derived:

$$
0 \leq E[V(t)] \leq\left(V(0)-\frac{D_{n}}{d_{0}}\right) e^{-a_{0} t}+\frac{D_{n}}{d_{0}} .
$$

Thus, the inequality,

$$
E\left[\left|z_{1}\right|^{4}\right] \leq E[V(t)] \leq 8 \frac{D_{n}}{d_{0}},
$$

holds for $\forall t>T_{1}$ with

$$
T_{1}=\max \left\{0,\left(\frac{1}{d_{0}}\right) \ln \left(\left[\frac{d_{0} v(0)}{D_{n}}\right]\right)\right\} .
$$

The proof is completed.

Remark 1. The controller in (54) contains neural networks structure, which will cause tedious calculation in practical application. Nevertheless, the inequality $0<S_{i}^{T}\left(Z_{i}\right) S_{i}\left(Z_{i}\right) \leq 1$ should also be emphasized, and it can be obtained from the theories in [46].

From Remark 1, the base functions in controller (54) can be conveniently deleted to further improve the design. Then, we give the following corollary.

Corollary 1. For $1 \leq i \leq n, k \in L$, we assume that the functions $f_{k, i}\left(\bar{\xi}_{i}\right)$ are, instead of by neural networks system, within bounded error $\varepsilon_{i}$. Consider the switching strategy among observer (11), the following are the intermediate virtual and the actual controllers:

$$
\begin{aligned}
& \alpha_{1}=-x_{1} z_{1}-\frac{3}{4} z_{1}-\frac{\widehat{\Theta}_{1}}{2 a_{1}^{2}} z_{1}^{3}-\frac{3(n-1)}{4} z_{1}\left(\varphi_{1} \varphi_{1}^{T}\right)^{2}, \\
& \alpha_{i}=-x_{i} z_{i}-\frac{3}{4} z_{i}-\frac{\widehat{\Theta}_{i}}{2 a_{i}^{2}} z_{i}^{3}, \quad 2 \leq i \leq n-1, \\
& u_{k}=-x_{n} z_{n}-\frac{3}{4} z_{n}-\frac{\widehat{\Theta}_{n}}{2 a_{n}^{2}} z_{n}^{3} .
\end{aligned}
$$

The adaptive laws denote as follows:

$$
\dot{\widehat{\Theta}}_{i}=\frac{r}{2 a_{i}^{2}} z_{i}^{6}-\varrho_{i} \widehat{\Theta}_{i}, \quad 1 \leq i \leq n .
$$

Then, the semiglobal boundedness of all signals in the closed-loop structure can be established under bounded initial values, and an arbitrary switching rate satisfies $\tau_{a}>\left(\log \left(\mu / a_{0}\right)\right)$. Meanwhile, we can obtain the arbitrarily small output with some suitable parameters.

Proof. This proof is rather similar to Theorem 1; therefore, we omit the procedure here.

\section{Simulation Example}

We will construct a numerical simulation example to verify the effectiveness of the proposed controllers. The following 
two stochastic switched second-order systems are listed as follows.

System (1) is defined as

$$
\begin{aligned}
\mathrm{d} x_{1} & =\left(g_{1,1}\left(\bar{x}_{1}\right) x_{2}+f_{1,1}(x)+h_{1,1}(x)\right) \mathrm{d} t+\psi_{1,1}^{T}(x) \mathrm{d} \omega, \\
\mathrm{d} x_{2} & =\left(u(v)+f_{2,1}(x)+h_{2,1}(x)\right) \mathrm{d} t+\psi_{2,1}^{T}(x) \mathrm{d} \omega, \\
y & =x_{1},
\end{aligned}
$$

where the switched signal $p=1$, and the nonlinear functions are

$$
\begin{aligned}
g_{1,1}\left(\bar{x}_{1}\right) & =0.5 \sin \left(x_{1}\right), \\
f_{1,1}(x) & =1-\cos \left(x_{1} x_{3}\right)+x_{2}+x_{3}, \\
h_{1,1}(x) & =-x_{3}(t) \sin \left(0.5 x_{1}(t) x_{3}(t)\right), \\
\psi_{1,1}(x) & =0.5 x_{2}(t) \cos \left(x_{1}(t)\right), \\
f_{2,1}(x) & =1+\cos \left(x_{2} x_{3}\right)+x_{3}, \\
h_{2,1}(\xi) & =0.5 \xi_{1}(t) \xi_{3}(t)-\xi_{3}(t), \\
\psi_{2,1}(\xi) & =\xi_{2}(t) \sin \left(\xi_{1}(t)\right) .
\end{aligned}
$$

System (2) is defined as

$$
\begin{aligned}
\mathrm{d} x_{1} & =\left(g_{1,2}\left(\bar{x}_{1}\right) x_{2}+f_{1,2}(x)+h_{1,2}(x)\right) \mathrm{d} t+\psi_{1,2}^{T}(x) \mathrm{d} \omega, \\
\mathrm{d} x_{2} & =\left(u(v)+f_{2,2}(x)+h_{2,2}(x)\right) \mathrm{d} t+\psi_{2,2}^{T}(x) \mathrm{d} \omega, \\
y & =x_{1},
\end{aligned}
$$

where the switched signal $p=2$, and the nonlinear functions are

$$
\begin{aligned}
g_{1,2}\left(\bar{x}_{1}\right) & =\sin \left(x_{1} x_{2}\right), \\
f_{1,2}(x) & =1-x_{1}^{2} x_{3}+x_{3}, \\
h_{1,2}(x) & =x_{3}(t) \cos \left(x_{1}(t) x_{3}(t)\right), \\
\psi_{1,2}(x) & =x_{2}(t) \sin \left(x_{1}(t)\right), \\
f_{2,2}(x) & =x_{2}^{2} x_{3}+x_{1}, \\
h_{2,2}(x) & =x_{3}(t) \sin \left(x_{1}(t)\right), \\
\psi_{2,2}(x) & =x_{2}(t) x_{1}(t) \cos \left(x_{2}(t)\right) .
\end{aligned}
$$

The input saturation is defined as

$$
u(v(t))=\operatorname{sat}(v(t))= \begin{cases}3 \operatorname{sign}(v(t)) \varepsilon_{0}, & |v(t)| \geq 3, \\ v(t), & |v(t)|<3 .\end{cases}
$$

Furthermore, the reference signal is given as $y_{d}=0.4(\sin (t)+\sin (0.5 t))$, and the designed parameters are chosen to be $\tau=0.2, k_{1}=k_{2}=1.3, \gamma_{1}=\gamma_{2}=1.4$, and $\sigma_{1}=\sigma_{2}=0.2$. The initial conditions are $x(0)=\left[\begin{array}{lll}0.01 & 0.03 & 0.15\end{array}\right]^{T}$ and $\widehat{\Theta}_{0}=\left[\begin{array}{lll}0.02 & 0.03 & 0.01\end{array}\right]^{T}$.

The simulation results are illustrated in Figures 1-5, respectively. Figure 1 demonstrates control output and target. Figure 2 gives the trajectories of adaptive parameter $\theta_{1}$ and $\theta_{2}$. Figure 3 shows the control input signal $u$. The input signal $u$ is asymptotically stable with the change of

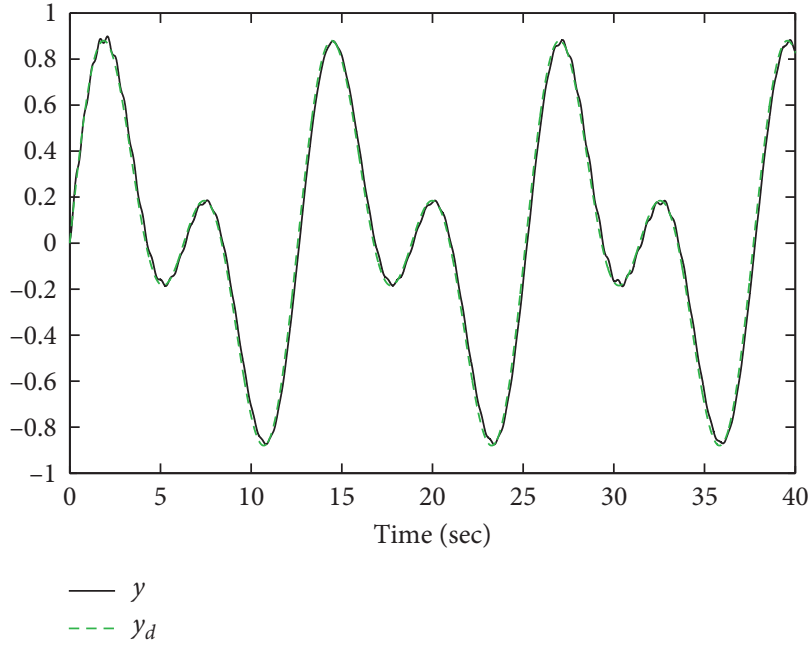

Figure 1: The response of the variables $y$ and $y_{d}$.

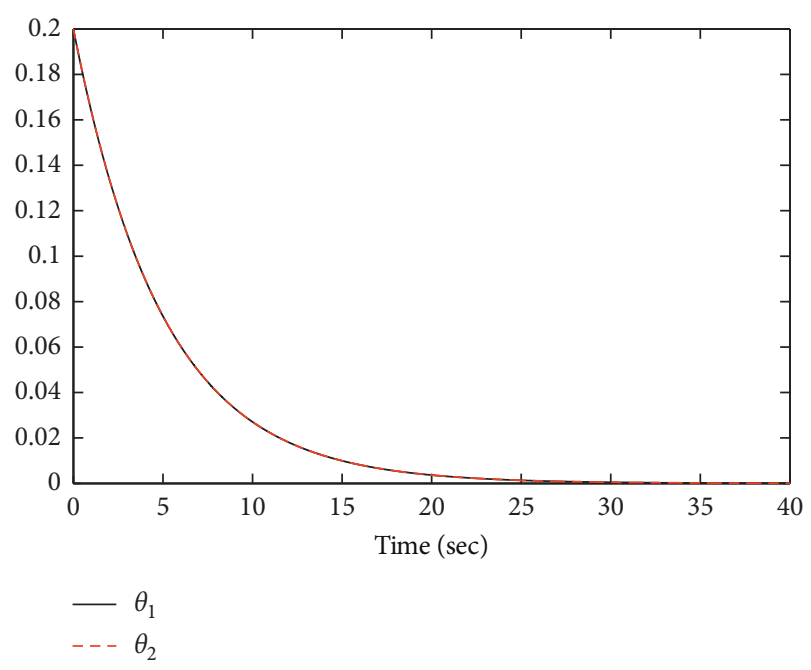

Figure 2: The response of the adaptive laws $\theta_{1}, \theta_{2}$.

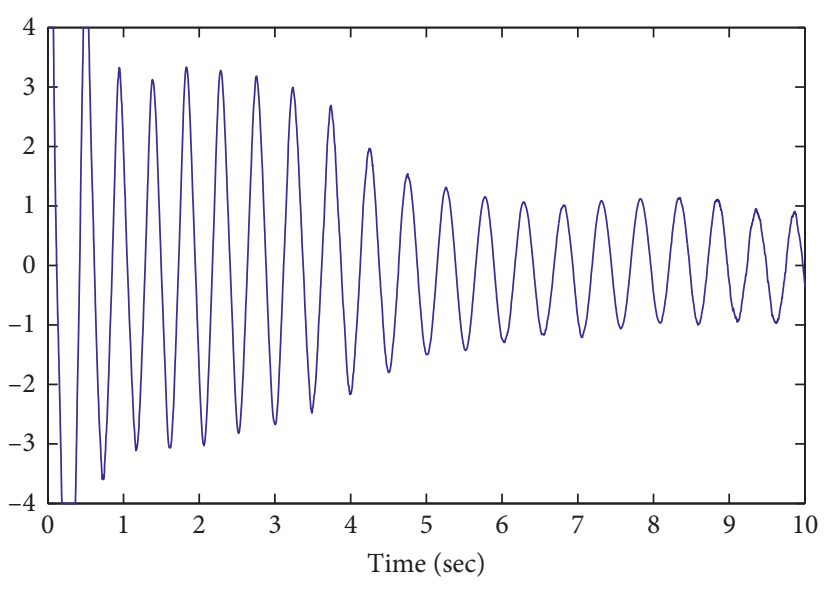

Figure 3: The response of the control input signal $u$. 


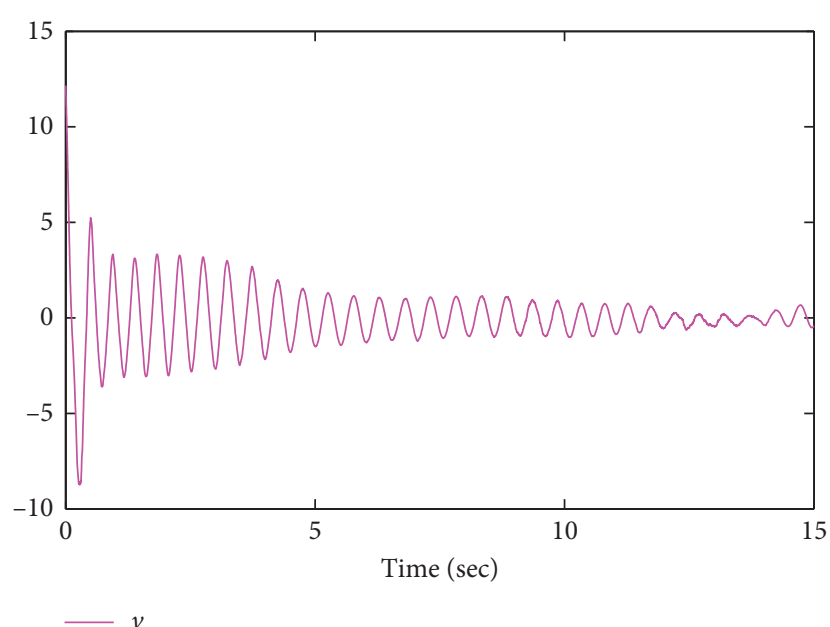

Figure 4: The response curves of state variable $v$.

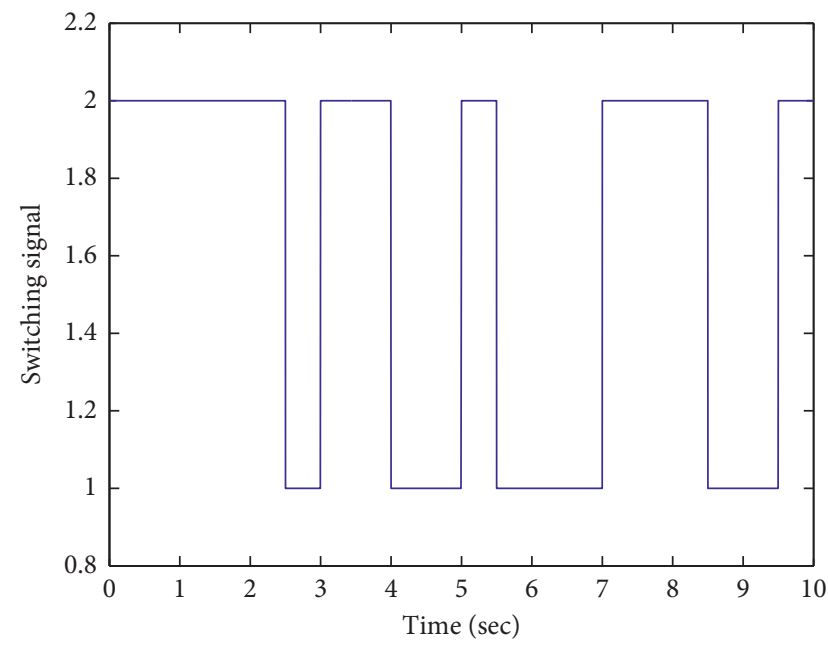

FIGURE 5: The response of the giving switching signal $\sigma(t)$.

time. Figure 4 shows the response curves of state variable $v$, and it can be drawn that $v$ converge to zero after a few seconds. Figure 5 shows the response curves of the giving switching signal $\sigma(t)$. From the above five figures, it can be seen that all signals in the closed-loop structure are bounded and the states can track the given reference signals. Based on the simulation results, we can conclude that the proposed control scheme is effective for the switched system.

\section{Conclusion}

An actual operating system is complex. When it is dominated by multiple switched subsystems with input saturation, external interference, nonstrict feedback, nonlinearity, and randomness, it is a difficult problem how to control it stably. This work comprehensively considers the influence of all the above factors, attempts to design a new switching controller and an adaptive neural scheme, combining with neural network structure, variable separation technology, reverse step method, Itô formula, and the auxiliary signal, gives the control design conditions of antisaturation and avoiding signal attenuation, and achieves the asymptotic stability performance of the closed-loop system. There are two difficulties in this paper, one is that the switching functions are unknown and nonstrict feedback, and the neural network structure is an effective function approximator at present. Therefore, we chose it to estimate these functions. The other is that how to design the switching strategy and controller to resist the saturation when the subsystem switches; to deal with this problem, we introduced a set of auxiliary system. Furthermore, we introduce a neural controller into the control scheme to track the variation of the error. The controller is designed based on the improved backstepping technique. It is proved that all the signals are bounded with some suitable parameters, and the trajectory error converges to zero.

\section{Data Availability}

The data used to support the findings of this study are included within the article.

\section{Conflicts of Interest}

The authors declare that they have no conflicts of interest.

\section{Acknowledgments}

This work was supported by the National Natural Science Foundation of China (11871117 and 61976027) and Natural Science Foundation of Liaoning Province of China (20180551262 and LQ2019008).

\section{References}

[1] S. Yin, H. Gao, J. Qiu, and O. Kaynak, "Descriptor reducedorder sliding mode observers design for switched systems with sensor and actuator faults," Automatica, vol. 76, pp. 282-292, 2017.

[2] H. Li, Z. Feng, X. Li, L. Liu, and B. Niu, "Decentralized adaptive approximation-based fuzzy output-feedback control of uncertain switched stochastic interconnected nonlinear systems," Journal of the Franklin Institute, vol. 354, no. 16, pp. 7306-7325, 2017.

[3] L. Zhang, S. Zhuang, and R. D. Braatz, "Switched model predictive control of switched linear systems: feasibility, stability and robustness," Automatica, vol. 67, pp. 8-21, 2016.

[4] B. Wang and Q. Zhu, "Stability analysis of semi-Markov switched stochastic systems," Automatica, vol. 94, pp. 72-80, 2018.

[5] X. Su, P. Shi, L. Wu, and Y.-D. Song, "Fault detection filtering for nonlinear switched stochastic systems," IEEE Transactions on Automatic Control, vol. 61, no. 5, pp. 1310-1315, 2016.

[6] H. Q. Wang, X. P. Peter Liu, J. L. Bao, X. J. Xie, and S. Li, "Adaptive neural output-feedback decentralized control for large-scale nonlinear systems with stochastic disturbances," IEEE Transactions on Neural Networks and Learning Systems, vol. 31, no. 3, pp. 972-983, 2019.

[7] H. R. Karimi, "Robust delay-dependent $H_{\infty}$ control of uncertain time-delay systems with mixed neutral, discrete, and distributed time-delays and Markovian switching 
parameters," IEEE Transactions on Circuits and Systems I: Regular Papers, vol. 58, no. 8, pp. 1910-1923, 2011.

[8] H. Q. Wang, P. X. P. Liu, X. D. Zhao, and X. P. Liu, "Adaptive fuzzy finite-time control of nonlinear systems with actuator faults," IEEE Transactions on Cybernetics, vol. 50, no. 5, pp. 1786-1797, 2019.

[9] H. Wang and Q. Zhu, "Finite-time stabilization of high-order stochastic nonlinear systems in strict-feedback form," Automatica, vol. 54, pp. 284-291, 2015.

[10] H. Wang and Q. Zhu, "Global stabilization of stochastic nonlinear systems via $\mathrm{C} 1$ and $\mathrm{C}$ controllers," IEEE Transactions on Automatic Control, vol. 62, no. 11, pp. 5880-5887, 2017.

[11] H. Ma, H. Li, H. Liang, and G. Dong, "Adaptive fuzzy eventtriggered control for stochastic nonlinear systems with full state constraints and actuator faults," IEEE Transactions on Fuzzy Systems, vol. 27, no. 11, pp. 2242-2254, 2019.

[12] T. S. Hu, Z. L. Sun, and Y. Z. Lin, Control Systems with Actuator Saturation Analysis and Design, Birkhauser, Basel, Switzerland, 2003.

[13] G. Grimm, J. Hatfield, I. Postlethwaite, A. R. Teel, M. C. Turner, and L. Zaccarian, "Antiwindup for stable linear systems with input saturation: an LMI-based synthesis," IEEE Transactions on Automatic Control, vol. 48, no. 9, pp. 15091525, 2003.

[14] E. F. Mulder, M. V. Kothare, and M. Morari, "Multivariable anti-windup controller synthesis using linear matrix inequalities," Automatica, vol. 37, no. 9, pp. 1407-1416, 2001.

[15] H. Wang, S. Liu, and X. Yang, "Adaptive neural control for non-strict-feedback nonlinear systems with input delay," Information Sciences, vol. 514, pp. 605-616, 2020.

[16] D. Cui, B. Niu, H. Wang, and D. Yang, "Adaptive neural tracking control of nonlinear stochastic switched non-lower triangular systems with input saturation," Neurocomputing, vol. 364, pp. 192-202, 2019.

[17] Y. X. Li and G. H. Yang, “Adaptive fuzzy decentralized control for a class of large-scale nonlinear systems with actuator faults and unknown dead zones," IEEE Transactions on Systems, vol. 47, no. 5, pp. 729-740, 2016.

[18] B. Niu, M. Liu, and A. Li, "Global adaptive stabilization of stochastic high-order switched nonlinear non-lower triangular systems," Systems \& Control Letters, vol. 136, Article ID 104596, 2020.

[19] Q. Zhu and H. Wang, "Output feedback stabilization of stochastic feedforward systems with unknown control coefficients and unknown output function," Automatica, vol. 87, pp. 166-175, 2018.

[20] S. Tong, C. Liu, and Y. Li, "Fuzzy-adaptive decentralized output-feedback control for large-scale nonlinear systems with dynamical uncertainties," IEEE Transactions on Fuzzy Systems, vol. 18, no. 5, pp. 845-861, 2010.

[21] Y. Li, S. Tong, and T. Li, "Composite adaptive fuzzy output feedback control design for uncertain nonlinear strict-feedback systems with input saturation," IEEE Transactions on Cybernetics, vol. 45, no. 10, pp. 2299-2308, 2015.

[22] H. Li, L. Wang, H. Du, and A. Boulkroune, "Adaptive fuzzy tracking control for strict-feedback systems with input delay," IEEE Transactions on Fuzzy Systems, vol. 25, no. 3, pp. 642$652,2016$.

[23] Q. Zhu, "Stabilization of stochastic nonlinear delay systems with exogenous disturbances and the event-triggered feedback control," IEEE Transactions on Automatic Control, vol. 64, no. 9, pp. 3764-3771, 2019.
[24] Q. Zhou, H. Li, C. Wu, L. Wang, and C. K. Ahn, "Adaptive fuzzy control of nonlinear systems with unmodeled dynamics and input saturation using small-gain approach," IEEE Transactions on Systems, Man and Cybernetics: Systems, vol. 47, no. 8, pp. 1979-1989, 2016.

[25] Z. Feng and P. Shi, "Two equivalent sets: application to singular systems," Automatica, vol. 77, pp. 198-205, 2017.

[26] B. Niu, Y. J. Liu, W. L. Zhou, H. T. Li, P. Y. Duan, and J. Q. Li, "Multiple Lyapunov functions for adaptive neural tracking control of switched nonlinear nonlower-triangular systems," IEEE Transactions on Cybernetics, vol. 50, no. 5, pp. 18771886, 2019.

[27] H. Li, L. Bai, L. Wang, Q. Zhou, and H. Wang, "Adaptive neural control of uncertain nonstrict-feedback stochastic nonlinear systems with output constraint and unknown dead zone," IEEE Transactions on Systems, Man, and Cybernetics: Systems, vol. 47, no. 8, pp. 2048-2059, 2017.

[28] B. Niu, P. Zhao, J. D. Liu, H. J. Ma, and Y. J. Liu, "Global adaptive control of switched uncertain nonlinear systems: an improved MDADT method," Automatica, vol. 115, 2020.

[29] B. Niu, D. Wang, H. Li, X. Xie, N. D. Alotaibi, and F. E. Alsaadi, "A novel neural-network-based adaptive control scheme for output-constrained stochastic switched nonlinear systems," IEEE Transactions on Systems, Man, and Cybernetics: Systems, vol. 49, no. 2, pp. 418-432, 2019.

[30] H. Li, L. Bai, Q. Zhou, R. Lu, and L. Wang, "Adaptive fuzzy control of stochastic nonstrict-feedback nonlinear systems with input saturation," IEEE Transactions on Systems, Man, and Cybernetics: Systems, vol. 47, no. 8, pp. 2185-2197, 2017.

[31] H. Wang and Q. Zhu, "Adaptive output feedback control of stochastic nonholonomic systems with nonlinear parameterization," Automatica, vol. 98, pp. 247-255, 2018.

[32] L. Cao, H. Li, N. Wang, and Q. Zhou, "Observer-based eventtriggered adaptive decentralized fuzzy control for nonlinear large-scale systems," IEEE Transactions on Fuzzy Systems, vol. 27, no. 6, pp. 1201-1214, 2018.

[33] H. Wang and Q. X. Zhu, "Global stabilization of a class of stochastic nonlinear time-delay systems with SISS inverse dynamics," IEEE Transactions on Automatic Control, 2020.

[34] J. Xia, J. Zhang, W. Sun, B. Zhang, and Z. Wang, "Finite-time adaptive fuzzy control for nonlinear systems with full state constraints," IEEE Transactions on Systems, Man, and Cybernetics: Systems, vol. 49, no. 7, pp. 1541-1546, 2019.

[35] Y.-J. Liu, S. Tong, C. L. P. Chen, and D.-J. Li, "Neural controller design-based adaptive control for nonlinear MIMO systems with unknown hysteresis inputs," IEEE Transactions on Cybernetics, vol. 46, no. 1, pp. 9-19, 2016.

[36] S. S. Ge and J. Zhang, "Neural-network control of nonaffine nonlinear system with zero dynamics by state and output feedback," IEEE Transactions on Neural Networks, vol. 4, pp. 900-918, 2003.

[37] X. Yu and X. J. Xie, "Output feedback regulation of stochastic nonlinear systems with stochastic iiss inverse dynamics," IEEE Transactions on Automatic Control, vol. 2, pp. 304-320, 2010.

[38] C. L. P. Chen, G.-X. Wen, Y.-J. Liu, and Z. Liu, "Observerbased adaptive backstepping consensus tracking control for high-order nonlinear semi-strict-feedback multiagent systems," IEEE Transactions on Cybernetics, vol. 46, no. 7, pp. 1591-1601, 2016.

[39] C. L. P. Chen, Y. J. Liu, and G. X. Wen, "Fuzzy neural network-based adaptive control for a class of uncertain nonlinear stochastic systems," IEEE Transactions on Cybernetics, vol. 44, no. 5, pp. 583-593, 2014. 
[40] Q. Zhou, P. Shi, and H. Liu, "Neural-network-based decentralized adaptive output-feedback control for large-scale stochastic nonlinear systems," IEEE Transactions on Cybernetics, vol. 6, pp. 1608-1619, 2012.

[41] L. Cao, Q. Zhou, G. Dong, and H. Li, "Observer-based adaptive event-triggered control for nonstrict-feedback nonlinear systems with output constraint and actuator failures," IEEE Transactions on Systems, Man and Cybernetics: Systems, vol. 9842, pp. 1-12, 2019.

[42] Z. Zhang, H. Liang, C. Wu, and C. K. Ahn, "Adaptive eventriggered output feedback fuzzy control for nonlinear networked systems with packet dropouts and random actuator failure," IEEE Transactions on Fuzzy Systems, vol. 99, pp. 608-619, 2019.

[43] Y. Li, Z. Ma, and S. Tong, "Adaptive fuzzy output-constrained fault-tolerant control of nonlinear stochastic large-scale systems with actuator faults," IEEE Transactions on Cybernetics, vol. 47, no. 9, pp. 2362-2376, 2017.

[44] H. E. Psillakis and A. T. Alexandridis, "NN-based adaptive tracking control of uncertain nonlinear systems disturbed by unknown covariance noise," IEEE Transactions on Neural Networks, vol. 18, no. 6, pp. 1830-1835, 2007.

[45] J. Xia, J. Zhang, J. Feng, Z. Wang, and G. Zhuang, "Command filter-based adaptive fuzzy control for nonlinear systems with unknown control directions," IEEE Transactions on Systems, Man and Cybernetics: Systems, vol. 109, pp. 1-9, 2019.

[46] L.-X. Wang and J. M. Mendel, "Fuzzy basis functions, universal approximation, and orthogonal least-squares learning," IEEE Transactions on Neural Networks, vol. 3, no. 5, pp. 807-814, 1992. 